\title{
Dispersion Characteristics of Double-Corrugated Rectangular Waveguide for Terahertz Vacuum Devices
}

\author{
Yulu $\mathrm{Hu}^{1}$, Quan $\mathrm{Hu}^{1}$, Mauro Mineo ${ }^{2}, \mathrm{Li} \mathrm{Li}^{3}$, Francesco Napoli ${ }^{2}$, Bin $\mathrm{Li}^{1}$, \\ Xiaofang Zhu ${ }^{1}$, Jianqing $\mathrm{Li}^{1}$, $\mathrm{Li} \mathrm{Xu}^{1}$, Tao Huang ${ }^{1}$ Xiaolin $\mathrm{Jin}^{1}$ and Claudio Paoloni ${ }^{2}$ \\ ${ }^{1}$ University of Electronic Science and Technology of China, Sichuan, 6110054, China \\ ${ }^{2}$ Lancaster University, Lancaster, LA1 4YW, UK \\ ${ }^{3}$ Beijing Vacuum Electronics Research Institute, China
}

\begin{abstract}
An analytical study on the dispersion of the double-corrugated waveguide for $\mathrm{THz}$ vacuum devices is presented. The boundary element method (BEM) is introduced to improve the accuracy of the dispersion. The results are compared with the 3D electromagnetic simulations.
\end{abstract}

\section{INTRODUCTION}

Oources and amplifiers at submillimeter and terahertz $\checkmark$ frequency are needed to enable attractive applications in fields as imaging, security or medicine. The double-corrugated waveguide (DCW), which can be easily realized by modern microfabrication techniques, has been studied to be implemented in BWOs [1] and TWTs [2]. The analysis was performed so far by 3D electromagnetic simulations due to the lack of a dedicated electromagnetic model.

In this paper, the dispersion characteristics of the DCW have been modeled by analytic equations to provide a fast electromagnetic computational tool. The schematic in Fig. 1 and Fig.2 are considered for the analysis.

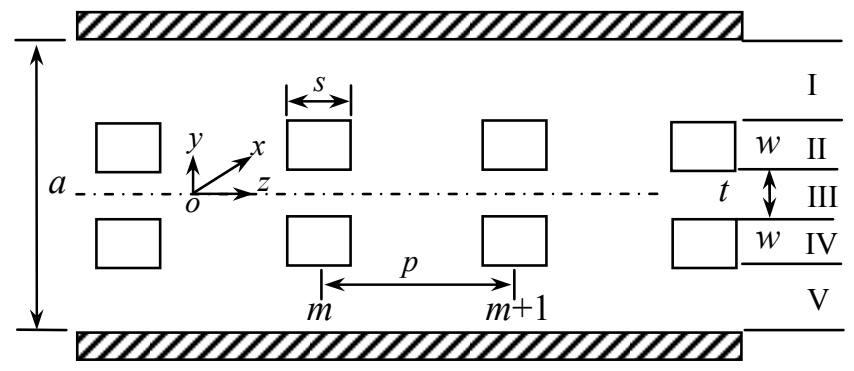

Fig.1 Top view of the DCW

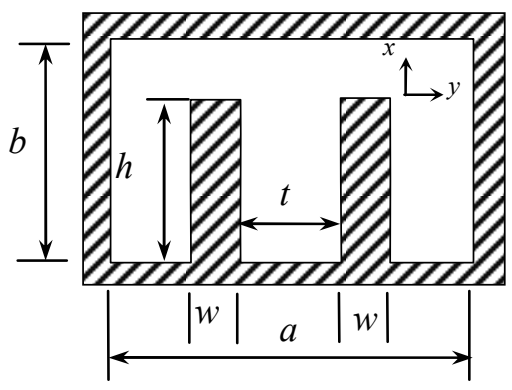

Fig.2 Front view of the DCW.

\section{MODEL}

The first TEM mode is assumed to propagate along the two pillars in the x-direction. The Floquet theorem and standing rectangular waveguide field expressions are applied in part I,
III and V. While the regions II and IV are assumed with a uniform field. The fields in YZ plane can be obtain by the applying boundary condition on the lateral metal walls and the integral condition in the discontinuity between the different regions. The admittance between the equivalent current and voltage is then established.

The admittance $Y_{0}$ can be written as:

$$
\begin{aligned}
Y_{0}(\phi)=2 \sqrt{\frac{\varepsilon_{0}}{\mu_{0}}} & \sin \frac{\phi}{2}\left\{\frac{2 w}{p-s} \sin \frac{\phi}{2}+\frac{s}{p} \sum_{n=-\infty}^{\infty}(-1)^{n} \frac{\sin \frac{\beta_{n}(p-s)}{2}}{\frac{\beta_{n}(p-s)}{2}}\right. \\
& \left.\cdot \frac{\sin \frac{\beta_{n} s}{2}}{\frac{\beta_{n} s}{2}}\left[\operatorname{cth} \beta_{n}\left(\frac{a-t}{2}-w\right)+\operatorname{cth} \beta_{n} t-\frac{1}{\operatorname{sh} \beta_{n} t}\right]\right\}
\end{aligned}
$$

$\varepsilon_{0}$ and $\mu_{0}$ are the permittivity and permeability respectively. $\underline{n}$ indicates the space harmonic number.

The equivalent voltage and current on the pillar $m$ can be written as

$$
\left.\begin{array}{l}
V_{m}(x)=[A \cos k x+B \sin k x] e^{-j m \phi} \\
I_{m}(x)=j Y_{0}(\phi)[B \cos k x-A \sin k x] e^{-j m \phi}
\end{array}\right\}
$$

Here $\phi$ is the phase of the period, $A$ and $B$ are the coefficients.

Given the equivalent voltage to be zero at the bottom of the pillar and capacitance condition between the equivalent current and equivalent voltage on the top of the pillars, the dispersion equation can finally be obtained as

$$
\begin{gathered}
Y_{0}(\phi)=D(f) \\
D(f)=\omega C \tan k h
\end{gathered}
$$

where $\omega$ is the angular frequency and $k=\omega / c$ for TEM wave, where $c$ is the speed of light.

The capacitance $C$, between the top surface of the pillars and the metal cover, is obviously different compared with the value of infinite parallel-plate capacitance when $(b-h)>w$ or $(b-h)>s$, which will significantly affect the accuracy of the dispersion and the frequency accuracy on the right term of the Eqn. (2). To solve the issue the boundary element method (BEM) is introduced to derive $C$ by solving the discrete surface charge distribution keeping the uniform voltage distribution on two the metal and pillar surface [3].

The voltage $u$ on the surface can be written as follows

$$
u(\vec{r})=\frac{1}{4 \pi} \int_{\Omega_{M}} \frac{1}{\left|\vec{r}-\vec{r}^{\prime}\right|} q d \Omega_{M}+\frac{1}{4 \pi} \int_{\Omega_{P}} \frac{1}{\left|\vec{r}-\vec{r}^{\prime}\right|} q d \Omega_{P}
$$

$\Omega_{P}$ and $\Omega_{M}$ indicate the area of an element on the metal and pillar surface respectively. 
A matrix equation is established by each voltage on an element integral of the charge on the rest elements on two surfaces:

$$
[M]\{q\}=\{u\}
$$

$M$ is the matrix of the structure. $\{\cdot\}$ indicates a vector for charge or voltage distribution.

Finally, the introduction of the new capacitance (BEM) improves the accuracy of the dispersion compared to the case of parallel-plate capacitance.

\section{Results}

The dispersion for a DCW at $220 \mathrm{GHz}$ [2] has been calculated by solving Eqn.(3), assuming the waveguide dimension $a=530 \mu \mathrm{m}$ and $b=380 \mu \mathrm{m}$, the period $p=360 \mu \mathrm{m}$, the height of the pillar $h=210 \mu \mathrm{m}$, the width $w=80 \mu \mathrm{m}$, the thick $s=280 \mu \mathrm{m}$ and the space between pillars $t=150 \mu \mathrm{m}$.

Table I

THE PARAMETERS OF A STRUCTURE

\begin{tabular}{|c|c|}
\hline Parameters & Value $(\mu \mathrm{m})$ \\
\hline$a$ & 530 \\
\hline$b$ & 380 \\
\hline$p$ & 360 \\
\hline$h$ & 210 \\
\hline$w$ & 80 \\
\hline$s$ & 320 \\
\hline$t$ & 150 \\
\hline
\end{tabular}

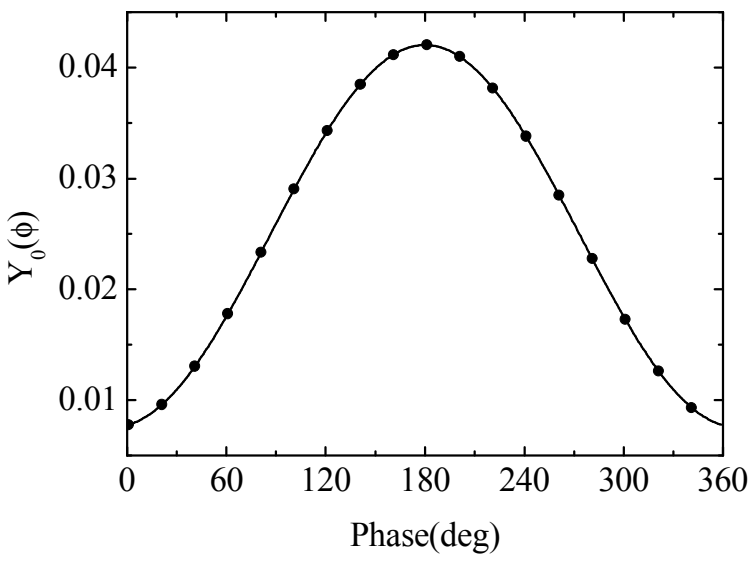

Fig. 3 The curve of $Y_{0}$ vs. phase of the period.

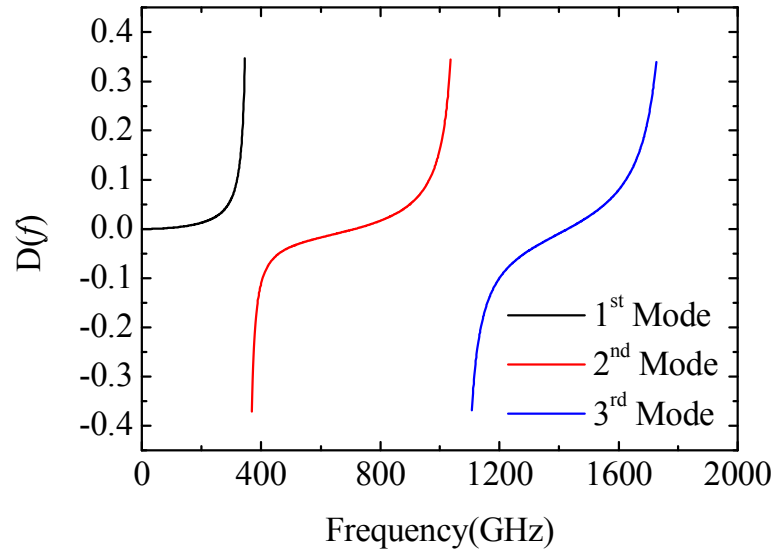

Fig.4 The curves of $D(f)$ of Eqn.(4) vs. frequency, which have numbers of modes.
The curve of $Y_{0}$ as a function of the phase is shown in Fig.3. The curves of $D$ of Eqn.(4) as a function of the frequency is shown in Fig.4. It can be noted the different modes function because of the tangent function.

The dispersion of the fundamental mode is considered to compare the two described approaches with 3D electromagnetic simulation performed by CST- MWS (Fig 5). The analytic model using the BEM computed capacitance has very good agreement with the $3 \mathrm{D}$ electromagnetic simulations.
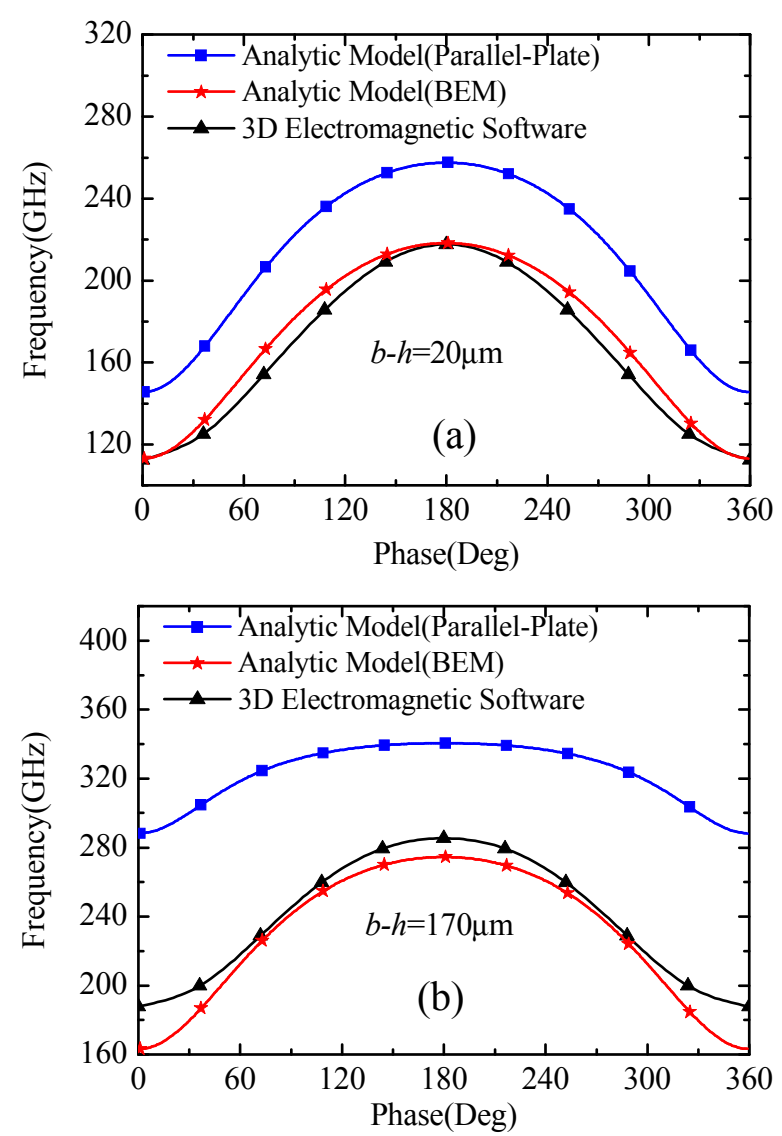

Fig.5 The dispersion comparison of the analytic model with parallel-plate capacitance and BEM capacitance and the 3D electromagnetic software assuming (a) $b-h=20 \mu \mathrm{m}$ and (b) $b-h=170 \mu \mathrm{m}$.

\section{CONCLUSION}

A analytical model for the dispersion of DCW has been developed and compared with 3D electromagnetic simulators. The use of the BEM capacitance gives good agreement with the $3 \mathrm{D}$ simulations.

\section{REFERENCES}

[1] Mauro Mineo and Claudio Paoloni, "Double-corrugated rectangular waveguide slow-wave structure for terahertz vacuum devices," IEEE Trans. on Electron Device, vol. 57, pp. 3169-3175, 2010.

[2] Paoloni, C.; Mineo, M., "Double Corrugated Waveguide for G-Band Traveling Wave Tubes," Electron Devices, IEEE Transactions on , vol.61, no.12, pp.4259,4263, Dec. 2014

[3] Hitoshi Nishiyama and Mitsunobu Nakamura, "Form and capacitance of parallel-plate capacitors," IEEE Trans. on Components, Packaging, and Manufacturing Technology-part A, vol. 17, pp. 477-484, 1994.

[4] A. F. Harvey. "Periodic and Guiding Structures at Microwave Frequencies," IRE Trans. on Microwave Theory and Techniques, vol. 8, pp.30-61, 1960.

[5] Brian D. McVey, Mark A. Basten and John H. Booske. "Analysis of Rectangular Waveguide-Gratings for Amplifier Applications," IEEE Trans. on Microwave Theory and Techniques, vol. 42, pp. 995-1003, 1994. 